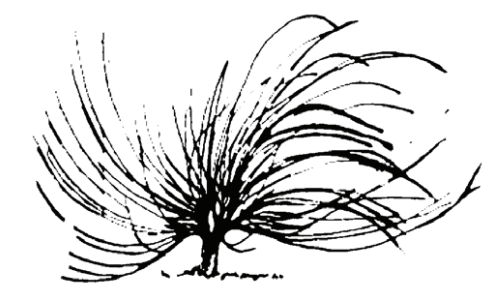

\title{
Hacia una nueva espiritualidad desde la pedagogía holística
}

\author{
Rocío Castillo Cedeño ${ }^{1}$ \\ Universidad Nacional, Costa Rica \\ Heredia, Costa Rica \\ rocíocastillocedeno@gmail.com
}

\begin{abstract}
Resumen
Este ensayo propone, desde una perspectiva holista, una reflexión crítica sobre la pedagogía como actividad vital que puede contribuir a la construcción de una sociedad más justa e inclusiva. Para ello, se presentan algunas apreciaciones fundamentales en la búsqueda de nuevos significados pedagógicos, siguiendo los enfoques de la exploración y la imaginación; lo lúdico y lo sensible; la autorregulación; la integración y la inclusión; la ecología y el desarrollo sostenible; y la complejidad.
\end{abstract}

Palabras clave: pedagogía holística, nuevos enfoques pedagógicos, espiritualidad.

\begin{abstract}
This paper proposes, from a holistic perspective, a critical reflection on pedagogy as a vital activity that can contribute to building a more just and inclusive society. For that purpose, some fundamental insights in the search for new pedagogical meanings are presented, according to approaches based on exploration and imagination; playfulness and sensitivity; self-regulation, integration and inclusion; ecology and sustainable development, and complexity.
\end{abstract}

Recibido: 30 de noviembre de 2011 - Aprobado: 30 de mayo de 2012

1 Doctora en Educación con mención pedagógica. Profesora de la División de Educación Básica, Centro de Investigación y Docencia en Educación, de la Universidad Nacional, Costa Rica. 
Keywords: holistic education, new educational approaches, spirituality.

L a humanidad vive tiempos difíciles, la preocupación por la racionalidad y la verdad absoluta han construido un planeta peligroso, de esa manera, se ha ido acumulando una enorme cantidad de armas químicas, atómicas, biológicas y hasta psicológicas.

La contaminación avanza a pasos agigantados, el flagelo del hambre condena a millones de personas en el mundo, la desigualdad predomina en todas las sociedades. La violencia produce muerte, terror y desesperación.

No se tiene la capacidad de predecir la totalidad de los problemas a los que se enfrentarán los seres humanos del futuro pero si tenemos el deber de empezar a gestar acciones que produzcan cambios significativos en el mundo.

Es evidente que la continuidad de la vida en la tierra está hoy amenazada, nos encontramos en un momento determinante que Capra (1982), denomina punto crucial, quizás porque es urgente el cambio y este debe darse ya, sin esperar.

Para ello, es necesario considerar la crisis, pero también, la capacidad constructiva del ser humano y las buenas obras y vibraciones, que día a día, se evidencian, pues si nos quedamos en la desilusión y el lamento, se desperdicia la energía positiva que se crea en el esfuerzo individual y colectivo, se debe enfocar el reto como una oportunidad y buscar en el espíritu interior la energía para trabajar de forma cooperativa.

Urge alejarnos de la rigidez atomizada y excluyente que ha predominado históricamente a través del paradigma mecanicista. Es fundamental, acercarnos a la humanidad, considerando los planteamientos de un paradigma holístico, que nos recuerdan que el Universo es sistémico, inclusivo, relacional y solidario.

Una visión que nos lleve a entender la espiritualidad como una ampliación y expansión de la conciencia y nos permita reconocemos como sistemas interconectados con otros sistemas. Entender que somos una unidad en la diversidad.

Así como lo señala Morín (2001, p. 72), "Hay una unidad humana. Hay una diversidad humana. Hay unidad en la diversidad, hay diversidad en la unidad humana..." 
Para Maturana(1999), lo que llamamos experiencias espirituales o místicas son experiencias de pertenencia o de comunidad en un ámbito más amplio que el de la realización personal. El fenómeno espiritual es un estado de conciencia un modo de vivir en relación con el otro.

Dentro de esta visión holística, el yo que creemos que somos no es un yo estático sino más bien dinámico, uno en continuo proceso de cambio, abierto, que va construyéndose y desconstruyéndose permanentemente, en la medida en que crece, evoluciona y se interrelaciona.

Entender esta perspectiva, implica abrir el espacio para que se desarrolle una mentalidad cósmica, donde la conciencia cooperativa es fundamental, donde todos como parte de un cosmos de interrelaciones realicemos acciones conjuntas que promuevan la vida. Así como propone el cantante y poeta Facundo Cabral (1990: p.15), "Si nos hubiéramos detenido en esta crisis a sentir, hubiéramos encontrado coincidencias, lo que tenemos en común, empezando por la vida. En tiempos tan homicidas y suicidas como éstos es más importante ser un hombre bueno que inteligente..."

Dentro de esta nueva espiritualidad, la conciencia cósmica es el punto medular, pues implica un nuevo pacto del ser humano con todos los seres que conforman el cosmos, implica una sinergia que nos une a todo, un impulso genuino por sentirse entremezclado en una danza integral, sentirse uno en medio de la diversidad.

Según Boff (2002, p.67), no puede desarrollarse una conciencia cósmica: "Si detrás de la ética no hay una mística, una espiritualidad, es decir, un nuevo pacto del ser humano con todos los demás seres, fundada en una nueva religación".

Según el autor una nueva religación es comprender que nada existe fuera de la relación, que todos tenemos una interioridad que al unirse con otras interioridades surge la espiritualidad, por ello, la espiritualidad no nace en la soledad, surge en lo que éste denomina Inter-retro-relación.

Considerando estos planteamientos, la conciencia cósmica se puede entender como:

La posibilidad de sentirse uno con el todo... Es la sensación de creer firmemente que cada cosa que haga por pequeña que sea, afecta a todo lo que me rodea. Es sentirse parte de un todo infinito que implica una frecuencia de energía, solidaridad, cooperación y amor. 
La conciencia por tanto, es personal y cósmica. Personal, pues surge en mi yo interior y cósmica cuando "es esencialmente relación hacia todos lados y en todas direcciones... estamos hechos del mismo material y somos frutos de la misma dinámica cósmica" Boff (2002, p. 75).

Aportando ideas similares, Gallegos (2000, p. 200) enriquece estos planteamientos señalando que cuando la conciencia está inmersa en un contexto de integración, interrelación y ligada profundamente a la espiritualidad, nos permite reconocernos como "parte de una gran familia humana y del universo en general, podemos hablar entonces del despertar de una conciencia cósmica, que permite sentir el mundo como parte de uno mismo".

Partiendo de las ideas anteriores, es primordial pensar en los espacios que pueden potenciar esta conciencia y quizás, uno de los más importantes es el pedagógico. Desde este espacio, se pueden realizar múltiples acciones y propiciar momentos claves para encontrarnos con la nueva espiritualidad.

Dentro de este contexto surgen muchas interrogantes: ¿cómo puede la pedagogía transformarse en una actividad vital, que permita al ser humano superarse al lado de los otros, promoviendo una sociedad más justa e inclusiva? ¿Cómo puede la escuela alejarse de la lógica mecanicista y acercarse a la holista? ¿Cómo erradicar los valores materialistas y dar paso a los valores trascendentales?.

Es difícil concretar la respuesta a estas preguntas y dentro de este ensayo quizás no se encuentren respuestas, mis pretensiones van más enfocadas a brindar algunas apreciaciones de lo que considero fundamental en la búsqueda de nuevos significados pedagógicos.

A continuación, se desarrollan diferentes ideas acerca de la pedagogía necesaria para acercarse a la nueva espiritualidad.

\section{Pedagogía de la exploración y la imaginación}

La concepción mecanicista de educación, nos ha llevado a considerar el aprendizaje como adquisición de conocimientos donde es indispensable la enseñanza. Pareciera que la premisa sería "si no se enseña no se aprende".

Al leer a Assmann (2002 p.35), nos encontramos con una propuesta muy rica: "la vida es esencialmente aprender... la esencia de estar vivo es sinónimo de estar interactuando, como aprendiente..." 
Esto permite pensar que la construcción del aprendizaje no es el resultado de ninguna enseñanza, es el resultado de la propia iniciativa del ser vivo. El conocimiento se construye progresivamente mediante acciones adaptativas, el ser vivo, en busca de aprender para sobrevivir.

Así como lo señala Assmann (2002 p. 105), “el conocimiento no es recibido de modo pasivo, mediante los sentidos o por transmisión, sino que es algo construido de manera activa por el sujeto conocedor".

Atendiendo las ideas de Assmann, H., estas situaciones de aprendizaje las brinda la vida pero pueden ser facilitadas por un espacio educativo rico en experiencias y un educador que cumpla una función mediadora.

En primera instancia es medular pensar en una Pedagogía de las preguntas y no de las respuestas, acercar a los(as) estudiantes a la investigación constante, activa y reflexiva. Para ello, es fundamental propiciar experiencias de aprendizaje que inviten a la observación, indagación y experimentación. Cosachov (2000, p. 37) nos recuerda de forma poética lo que es aprender: "Es quedarse prendido de la pregunta sin respuesta inmediata".

Los espacios de interacción deben organizarse de forma que faciliten la acción, el juego y el aprendizaje compartido.

Así como se plantea Hetland (2005 p. 88), refiriéndose al proyecto cero, se requiere una enseñanza para la comprensión, la cual implica la participación activa del estudiante en el proceso educativo. Consiste en incorporar en la cotidianidad constantes momentos de exploración.

Es necesario, que las experiencias de aprendizaje surjan de la propias producciones y vivencias de los estudiantes, no se debe crear una espacio artificial de aprendizaje... el aprendizaje debe ser la vida misma. Es urgente liberar a los estudiantes de la alineación, por ello, se considera la creatividad como base de la acción pedagógica.

Todo lo anterior, no se logra si la educación sigue reproduciendo los intereses de la ideología dominante, si la pedagogía no propone experiencias de valoración y toma de conciencia de las necesidades e intereses reales de los seres humanos y el cosmos en general.

"La pedagogía debe promover el descubrimiento y la apertura de horizontes, lo que supone una implicación en el mundo, sostenida por el interés, la curiosidad y el propósito personal de comprender y encontrar sentido". Miller (1990, p. 11).

Se requieren experiencias de aprendizaje que orienten a los estudiantes para la incertidumbre que nos plantea un mundo en constante cambio. 
Según Gelb (1999, p.11), "la tolerancia a la confusión” debe ser un rasgo característico de las personas altamente creativas". Es muy importante, propiciar una relación genuina entre educadores, estudiantes y sociedad en general, estimulando el juicio crítico y el desarrollo independiente y tolerante. El desarrollo de un espíritu sensible, libre, feliz y apasionado.

Lo anterior, solamente es posible lograrlo si la pedagogía destierra la rigidez, el dominio y poder que tiene la escuela. La escuela requiere cambiar... es urgente evitar situaciones educativas que dominen y enajenen al estudiante.

Así como, lo afirma Gelb (1999, p.72), "si bien todos empezamos la vida con una curiosidad insaciable, como la de Da Vinci, la mayoría aprendimos, al llegar a la escuela, que las respuestas eran más importantes que las preguntas. En la mayoría de los casos la educación escolar no desarrolla la curiosidad, ni el gusto por la ambigüedad, ni la habilidad de hacer preguntas".

Definitivamente, es esencial implementar una pedagogía que proponga a los(as) estudiantes, como Payán (2000), "lanzarse al vacío" para que puedan abrir sus alas hacia el aprendizaje.

\section{Pedagogía lúdica, amorosa y sensible}

Así como lo menciona Payán (2000 p. 22), es necesario "realizar un trabajo educativo desde el senti-pensar", esto implica una pedagogía crítica que permita la trascendencia y el autoconocimiento.

Calvo(2005, p. 8), indica que "la educación como la vida es un eterno fluir que si se escolariza se convierte en un proceso artificial, rígido, fragmentado, aburrido y poco significativo".

La educación debe ser chamánica, ofrecer como principal medicina el amor, el disfrute y el júbilo espiritual. Hacer del espacio educativo una experiencia lúdica, donde la risa y el juego sean la metodología por excelencia.

Lo fundamental es considerar que la situación ideal para aprender, es aquella en que la actividad sea tan agradable que el aprendiente la considere a la vez placer y vivencia. Hacer que cada momento pedagógico sea una fiesta.

La pedagogía debe propiciar un conjunto de experiencias de aprendizaje en las que el organismo tome parte, sin otra razón, que el 
placer en sí, lo cual implica que muchas de estas vivencias se conviertan en aprendizajes significativos para el estudiante.

Cuando un aprendizaje es significativo, resuena en la experiencia vital, así como lo señala Flores (1994, p.29): "Un aprendizaje significativo es un nuevo nodo de sentido que el alumno anuda con los hilos de su propia telaraña cognitiva y vital".

Las experiencias de aprendizaje que se propongan deben invitar a la meditación y la contemplación de la naturaleza y del cosmos en su totalidad, con el propósito de que los(as) estudiantes aprendan el valor de su vida y del todo. Es básico, atender los afectos, emociones y sentimientos: cuidar la conciencia cósmica,

Acercar a los estudiantes a experiencias que impliquen tocar, oler, ver, sentir, vibrar y emocionarse, es permitirles, crecer como seres artísticos y tomar conciencia del milagro de la vida.

Una niña de tres años me solicita que salgamos al patio porque está muy cansada, yo acepté la sugerencia, e invité a los(as) estudiantes a salir del salón y acostarse en el zacate, a la sombra de un árbol. La estudiante que realizó la petición de salir, se lanza con júbilo en el césped y observa con detenimiento las nubes y comienza a cantar:

"Hola nubes, hola sol donde está mi corazón" yo me acerqué a ella y esta me abrazó mientras expresaba lo siguiente: -vieras que feliz que estoy-, yo le pregunté -¿por qué?- Y ella me responde con otra pregunta ¿Usted está feliz acostándose en el zacate?...

Luego de este acontecimiento, había en el suelo 8 niños más, mirando y cantando al cielo. Luego de unos minutos otro niño se me acercó afirmando "que lindo es vivir así"...

Si una experiencia tan simple como esta, pudo generar tanta sensibilidad: ¿cuántas otras cosas se pueden hacer en los espacios escolares?.

"Es tiempo de salir del analfabetismo que nos propone una lógica separada de la belleza. Es hora de dejar de ser forzados por los datos y permitir que los signos del arte entre en nosotros..." Cosachov, 2000 p.103) 


\section{Pedagogía de la autorregulación}

Una pedagogía para la autorregulación promueve la desobediencia vital, entendida como la posibilidad de cuestionamiento, creación, fluidez de la vida, en definitiva auto organización.

Estoy segura de que muchas estrategias de aprendizaje saludables y autorreguladas se podrían fomentar si tenemos clara la relación mente, cuerpo y espíritu.

Estar orgullosos del cuerpo, apreciarlo y tratarlo con bondad, también, significa contemplar las maravillas de la existencia, respirar la espiritualidad del ser y el poder de la mente.

Representa, tener conciencia del cuerpo como algo sagrado, de la mente según Dossey (2004, p.278), "como infinita, la mente como inmortal".

Los estudiantes deben ser invitados a utilizar su mente, cuerpo y espíritu para promover la iniciativa e inteligencia en la exploración activa del entorno, porque solo, con el intercambio directo con la realidad es como se desarrolla la capacidad biológica que da lugar a la inteligencia. La nueva percepción según Assmann (2002 p.69), consiste en "tomar como punto de partida la interrelación compleja entre procesos vitales y procesos cognitivos" P.69.

Una pedagogía de la autorregulación propicia que el(la) estudiante se descubra a sí mismo(a) como persona individual y a la vez colectiva, favoreciendo las comparaciones vivenciales, la comunicación creativa y el control mutuo.

Considerando lo anterior, el error es una experiencia más valiosa que el acierto, pues a través de éste, hay múltiples posibilidades de establecer interconexiones de conocimientos y propiciar el aprendizaje.

El ambiente escolar debe evitar que los(as) estudiantes teman a la equivocación. "La vida misma es expresión sinérgica de ella. Si la vida es así, con mayor razón, lo será el jardín infantil, la escuela y la universidad. En otras palabras, los profesores y estudiantes deben ir a equivocarse a la escuela, antes que buscar la respuesta estereotipada y segura pero rara vez comprendida" (Calvo, 1992, p. 16). 


\section{Pedagogía integradora e inclusiva: base de la espiritualidad}

"Existe una unidad en la diversidad y una diversidad en la unidad" Boff (2000, p. 75)

Cada estudiante tiene su propia historia, la cual se crea dentro de otro proceso macro-histórico, con raíces culturales permanentes, presentes y con posibilidades de modificarse, que marcan qué es lo que se puede conocer, qué es lo que se necesita saber para vivir en determinada sociedad y la escuela es el instrumento de transmisión por excelencia.

El reconocimiento de las diferencias no está ajeno a las relaciones de poder, que se legitiman socialmente y que deben considerarse en la escuela.

¿Qué papel debe cumplir la pedagogía para replantearse y reflexionar acerca de las relaciones de poder que se han legitimado históricamente en la escuela?

¿Cómo desterrar los estereotipos que han predominado y siguen predominando en las escuelas?

Para iniciar este transitar, es urgente evitar experiencias de aprendizaje que promueven la exclusión, toda situación escolar debe estar libre de prejuicios, los y las docentes tienen la tarea de revisar cuidadosamente las estrategias y recursos pedagógicos, usando un ojo crítico y sensible, que analice principalmente, los posibles estereotipos o relaciones dispares o discriminatorias.

Este ojo debe traspasar la estética y tomarse fuertemente de la ética y la cooperación. Las y los docentes no deben conformarse con un material "bonito" o una melodía pegajosa, es fundamental, ir más allá, localizando aquellos mensajes que puedan entorpecer el retorno a la cultura de la comprensión y cooperación.

Cuando pienso en lo anterior, me acerco a un ejemplo que siempre brindo a mis estudiantes de preescolar: la poesía de los deditos:

“este dedito se encontró un huevito (se saca el dedo meñique)

Este otro lo llevó a la casita (se señala el dedo anular)

Este otro lo cocinó (se señala el dedo medio)

Este otro lo peló (se enseña el dedo índice)

Y este fuerte gordo se lo comió (se señala el dedo pulgar)..." 
Si se lee con ojo crítico-sensible encontraremos que una poesía dactilar tan "tierna y sencilla" en apariencia, tiene más de una carga de relación de poder... "el más fuerte se comió el huevo, sin hacer nada"

Cuántas y cuántos docentes hoy realizan múltiples actividades y usan diferentes recursos que no son evaluados utilizando una óptica reflexiva.

Por ello, aún nos encontramos listas de asistencia por sexo, filas separadas (hombres y mujeres), las niñas limpiando las mesas y los niños apuntando en la pizarra a los que se "portan mal", cantando canciones como arroz con leche y jugando al ratón y el gato, sin determinar que en muchas de estas acciones, se están legitimando significativas relaciones de poder.

Es fundamental, que la pedagogía localice estas relaciones de poder y propicie el respeto por la diversidad, la escuela debe dialogar acerca del valor de las diferencias, principalmente de género, las cuales, deben atenderse considerando el respeto de la sincronicidad de la naturaleza: hombre y mujer: cada uno tiene un componente sexual que mantiene el equilibrio del cosmos.Es fundamental, que la escuela celebre día a día la diversidad.

El lenguaje juega un papel medular en la creación de significados, por ello, es muy importante utilizar un lenguaje inclusivo, que permita percibir en las diferencias, una forma de enriquecer el proceso. Es básico reinventar las palabras y promover nuevos significados inclusivos e integradores.

La forma de redefinirnos a nosotros y a los otros a través del lenguaje, de la conversación, es fundamental para el futuro de una nueva cultura. Cambiando el lenguaje construimos nuevas formas de interacción social y con esto formamos nuevas maneras de ser, de comprender cómo nos relacionamos, quiénes somos y para qué estamos en este mundo.

"el discurso y la lengua pueden utilizarse deliberadamente para obtener una mayor madurez cultural, una mayor justicia social" Iriguaray (1992)

Los distintos y múltiples accesos a la verdad nos obligan a una toma de conciencia, a cambiar el lenguaje jerárquico, verticalista, fundado en el par dominador-dominado correspondiente al mundo moderno, por un 
lenguaje de cooperación, de pensar y accionar de modo interdependiente: de pensar solidario, profundamente ligado a la espiritualidad.

Para lograr el cambio de lenguaje, es fundamental que en la escuela se establezcan relaciones amorosas cargadas de experiencias de aprendizaje cooperativas. Es importante, que los(as) estudiantes tengan la posibilidad de salir de sí mismos y colaborar con otras naturalezas individuales, este es el punto de partida de la supervivencia.

Así como lo plantea Assmann (2002, p.108), "hoy día el cambio epistemológico en la educación tiene que ver con la supervivencia y la calidad de vida del futuro en este planeta. Se trata de incluir, en el propio aprender, el aprender la vida y aprender el mundo, pensando en la construcción de un mundo donde quepan todos".

Es básico, compartir significados que nos acercan a un encuentro genuino con el otro, rompiendo las barreras paradigmáticas que impulsan las relaciones de poder.

Valorar la intuición, la poesía, la contemplación y la oración, aspectos básicos, de una sociedad matriarcal y claves esenciales, para ser parte de este mundo de valiosas diferencias. La conciencia cósmica se encuentra cuando observamos a los(as) niños(as) unir sus manos con los otros, sin distinciones ni barreras, cuando los escuchamos cantarle al sol y a la luna, cuando los vemos sonreír al árbol y dialogar con las nubes. Cuando se observa como ellos(as) entienden la unión del todo con el todo, las inter-retro-relaciones.

Disfrutar la diversidad y respetar la individualidad son condiciones necesarias para la eliminación de las brechas que separan a los seres humanos. Todo lo anterior, es parte integrante de la espiritualidad

y brinda sustento a la conciencia cósmica. A través de la espiritualidad los estudiantes se reconocen como parte de una gran familia humana.

\section{Pedagogía para la ecología y el desarrollo sostenible}

Cuidar la vida en todas sus manifestaciones, es una tarea básica de la pedagogía espiritual. 
La tierra está triste... debemos escuchar sus lamentos y hacer algo para arrullarla tiernamente, en ese arrullo nos estaremos acariciando mutuamente.

Es urgente atender los gritos de una tierra maltratada y desvalorizada, si no lo hacemos pronto estaremos llorando con ella...

Cuando pienso en desarrollo sostenible pienso en mi hijo... cada vez que imagino un mundo futuro devastado y poco habitable mi piel se eriza al visualizarlo a él y a todos(as) $\operatorname{los}($ as) niños(as) con los que comparto cada día.

Surge en mí una fuerza interior que me impulsa a pensar en como construir un mundo mejor para las generaciones futuras, pienso en mi responsabilidad como docente y el papel medular de la educación en esta construcción.

Considero, que es un buen impulso iniciar la toma de conciencia pensando en quienes amamos. Aquí surge una clave fundamental: el amor.

Es necesario, que la pedagogía propicie espacios donde se permita un crecimiento espiritual y humano que promueva el amor, no sólo, por los que conocemos y están cerca, sino también, por todos los seres que nos rodean, cerca o lejos. El punto de partida de esta misión es valorar la interconexión que existe entre todas las formas de vida.

La pedagogía debe permitir el desarrollo del reconocimiento de nuestra existencia conscientemente interactiva, interdependiente y dedicada a contribuir con cosmos.

La poderosa fuerza que debe impulsar el proceso de cambio hacia la sostenibilidad debe yacer en nuestra preocupación por los otros y en la compasión que impregne nuestras acciones.

¿Qué tipo de situaciones de aprendizaje son las que se requieren para lograr esta conciencia?

En definitiva, se debe partir de situaciones de aprendizaje que promuevan la cooperación, solidaridad, ternura y amor. Situaciones que faciliten la reflexión acerca del milagro de la vida. Aquí, partimos hacia otro aspecto primordial, el sentido de la vida.

Valorar la vida, es valorar todo... No podemos pretender asumir un desarrollo sostenible, sino amamos la vida. Debe surgir una reverencia y un respeto profundo por ésta. 
Los problemas ecológicos deben asumirse como una preocupación personal y canalizar todo esfuerzo, pensando en la armonía del futuro de la vida de la humanidad.

Por esta razón, la ética ecológica debe sentirse como un voto y un reto que nace en el corazón y que proporciona a nuestra vida un impulso para generar más vida.

Ante la crisis ecológica, es importante retomar algunas de las ideas expuestas por Gutiérrez (2003), las situaciones de aprendizaje que debe propiciar la pedagogía deben ir más allá de la denuncia. La educación debe permitir que los estudiantes conozcan y entiendan las causas y las estructuras sociales, económicas y políticas que han generado la destrucción. Ejerzan sus derechos y tomen una conciencia profunda de sus responsabilidades.

Todo lo anterior, solo se logra en la medida en que se promueva el desarrollo de valores cooperativos, la dimensión ética, la sensibilidad, imaginación, voluntad y creatividad. Esta, implica también, tomar conciencia de la tierra como ser viviente, tener la posibilidad de contemplación y meditación.

Con toda una energía positiva.... afirmo que podemos evolucionar espiritualmente, la pedagogía debe comprometerse con ello. Nuestra capacidad humana es infinita, por esto, nuestras posibilidades de creación serán mayores que las de destrucción.

\section{Pedagogía de la complejidad}

"El proceso educativo consiste en crear diversas relaciones sinérgicas gracias a las cuales las diferentes polaridades tales como saber-ignorancia, orden-caos, comprensión-confusión, se armonizan complementariamente y holísticamente en un constante fluir del uno y otro, sin antagonismos, donde lo distinto es acogido en su diferencia" (Calvo, 1993, p. 17)

La pedagogía de la complejidad consiste en plantear sistemas educativos muy abiertos, donde la diversidad presente tanto en los entornos como en los elementos y grupos, tiende a fluir y reflejarse en todas las dimensiones espacio temporales y de sentido. Esto se logra, según Miller (1990), "mediante procesos de mutua coexistencia, que 
al alejarse de las condiciones normales de equilibrio, y por tanto, del paradigma humano vigente, propician la autorregulación de redes de interconexión: en el conocimiento y en la vida".

En este sentido, es medular cambiar los conceptos tradicionales de adquisición de conocimiento, enseñanza, autoridad, disciplina, asignaturas, currículo, programas y evaluación. Por supuesto, la organización escolar que da forma material a todas esas concepciones.

Es fundamental, que este cambio no separe al estudiante del mundo, sino que lo incluya en él como partícipe creativo. Abandonando la ilusión de la objetividad. La complejidad está básicamente en atender la subjetividad individual y las interrelaciones que conforman la intersubjetividad.

Lo que significa, tratar el proceso educativo considerando la fluidez e interdependencia, la incertidumbre debe verse como algo normal en la que todos(as) estamos inmersos.

Para finalizar con este recorrido pedagógico desde la nueva espiritualidad, es clave resaltar lo que significa ser docente en este paradigma holístico.

\section{Profesar la docencia}

"...los maestros no adiestran señalan rutas y formas de búsqueda" Cosachov (2000, p.21).

Los(as) docentes acompañan y guían en el "ritual sagrado" del proceso pedagógico, promueven preguntas constantes e incentivan en la búsqueda de puentes para compartir significados.

Gallegos (1998, p.59) comparte estas ideas expresando que el(la) docente "requiere de una mezcla de sensibilidad artística y práctica científica".

Dentro de esta labor, lo más importante es el amor, por ello, Cossachov, (2000, p. 33) insiste en la relación de los amantes, una relación que los pedagogos y las pedagogas deben de tener con el todo... ser amantes de la vida y gozar de un encuentro genuino con los(as) estudiantes.

La profesión docente requiere de entrega y pasión que permita la expansión de lazos significativos, sensibles a las necesidades de los(as) estudiantes y a las señales que comunican... según Cossachov (2000, p 43), "estar en coincidencia". 
Si deseamos que nuestros educandos sean bailarines, cantores, dibujantes, poetas y sobre todo seres cooperativos solo debemos permitirles convivir con un cosmos cargado de luz, color, significados, sueños, matices pero sobre todo energía sagrada.

El(la) docente debe acercarse a los(as) aprendientes como un curandero de corazones, mentes y almas. Así como lo expresa Parras, J. citado en Payán, (2000, p.11) al describir la labor del indio, brujo o yerbatero: "éstos están allí para hacer cosquillas a nuestra conciencia universal y llenar de caricias nuestros cuerpos".

Ser docente es tener múltiples posibilidades de entender la nueva espiritualidad y transformar los espacios pedagógicos, en espacios de vida.

\section{Referencias bibliográficas}

Assmann, H. (2002) Placer y ternura en la educación. Madrid: Nancea.

Boff. L. (2002). Ecología grito de La Tierra: Grito de los pobres. Madrid: Trotta.

Briggs, J. y Peat, D. (1999) Las siete leyes del caos Nueva York: Grijalbo

Cabral, F. (1990) Ayer soñé que podía y hoy puedo.

Capra, F. (1985) El punto crucial. Barcelona: Integral.

Capra, F. (1998) La trama de la vida. Barcelona: Anagrama.

Calvo, C. (2005) Educación y naturaleza: el fluir de los procesos. Documento sin publicar.

Calvo, C. (1993) Un educador para el nuevo mundo. Chile: Proyecto multinacional de Educación Básica, Organización de Estados Americanos / Ministerio de Educación de Chile.

Cosachov, M. (2000) Entre el cielo y la tierra: un viaje por el mapa del conocimiento. Buenos Aires: Biblos.

Dossey, L. (2004) El poder curativo de la mente. México: Santillana.

Dossey, L. (1986) Tiempo, espacio y medicina. Barcelona: Cairos.

Delors, J. (1996) La Educación encierra un tesoro. Madrid: Santillana.

Ferreiro, R. (2000) Mediación y Educación. Buenos Aires: Kapeluz.

Flores, R. (1994) Hacia una pedagogía del conocimiento. Bogotá: Mc Graw Hill.

Gallegos, R. (2000) Educación holista. México: PAX.

Gallegos, R. (1997). El destino indivisible de la educación. Guadalajara: PAX

Gelb, M. (1999) Inteligencia Genial.Bogotá: Norma.

Gutiérrez, F. (2003) Ecopedagogía y Ciudadanía Planetaria. Heredia: Instituto Latinoamericano de Pedagogía de la Comunicación.

Gutiérrez. F., Prieto, D. (2002) Mediación Pedagógica. Universidad de San Carlos, Guatemala.

Gutiérrez, F. (2004) Pedagogía del aprendizaje: Guatemala.

Harris, M. Y Caníbales, R. (1986) Paradigmas. Canadá: OISE.

Irrigaría, L. (1992) Yo, tú, nosotras. España: Cátedra.

Kaku, M. (1998) Visiones. Cómo la ciencia revolucionará la materia, la vida y la mente en el siglo XXI. Madrid: Debate, S.A.

Lowenfeld, V. (1961) El niño y su arte. Buenos Aires: Kapeluz 
Lamas, M. Debate Feminista. Cuerpo y Política. (Sep 1994) Año 5, vol. 10

Maturana, Humberto (1999) Transformación en la convivencia. Chile: Editorial Universitaria.

Maturana, Humberto (1996) El árbol del conocimiento. Chile: Editorial Universitaria. Miller, J. (1996).The holistic curriculo. Canada: OISE.

Monard, J. (1987) Hacia allá. Bogotá: Mc Graw Hill.

Montessori, M. (2003) Educar para un nuevo mundo. Buenos Aires: Longseller.

Morin, E. (2003). La Humanidad de la Humanidad. Madrid: Cátedra.

Morin, E. (2001). Los siete saberes necesarios para la educación del futuro. España: Paidós.

Payàn, J.C. (2000) Lánzate al vacío. Colombia: Panamericana.

Verdú, V. (2003) El estilo del mundo. Madrid: Anagrama.

Wagensberg, J. (2002) Si la naturaleza es la respuesta, ¿cuál es la pregunta?. Barcelona: Matatemas. 